\title{
Prediction of early death after myocardial infarction in patients with reduced left ventricular ejection fraction. The search for new indications for cardioverter-defibrillator implantation (ICD)
}

\author{
Damian Pres ${ }^{1}$, Mateusz Tajstra', Przemysław Mitkowski², Aneta Ciślak', Kamil Bujak1, Jarosław Kaźmierczak³, \\ Maciej Sterliński ${ }^{4}$, Katarzyna Mizia-Stec ${ }^{5}$, Radosław Sierpiński ${ }^{6}$, Mariusz Gąsior ${ }^{1}$, Łukasz Szumowski ${ }^{6}$, \\ Zbigniew Kalarus $^{7}$ \\ ${ }^{1} 3^{\text {rd }}$ Department of Cardiology, Silesian Center for Heart Diseases, Faculty of Medical Sciences in Zabrze, Medical University of Silesia, Katowice, Poland \\ ${ }_{2}^{2} 1^{\text {st }}$ Department of Cardiology, Poznan University of Medical Sciences, Poznan, Poland \\ ${ }^{3}$ Department of Cardiology, Pomeranian Medical University, Szczecin, Poland \\ ${ }^{4}$ Department of Arrhythmia, National Institute of Cardiology, Warszawa, Poland \\ ${ }^{5} 1^{\text {st }}$ Department of Cardiology, Medical University of Silesia, Katowice, Poland \\ ${ }^{6}$ Department od Arrhythmia, Institute of Cardiology, Warszawa, Poland \\ ${ }^{7} 1^{\text {st }}$ Department of Cardiology, Congenital Heart Diseases, and Electrotherapy, Silesian Center for Heart Diseases, Zabrze, Poland
}

\author{
Correspondence to: \\ Damian Pres, MD, \\ Silesian Center for Heart Disease, \\ M Curie-Skłodowskiej 9 \\ 41-800 Zabrze, Poland, \\ phone: +48323733860 , \\ e-mail: damianpres@wp.pl \\ Copyright by the Author(s), 2021 \\ Kardiol Pol. 2021; \\ 79 (12): 1343-1352; \\ DOI: 10.33963/KP.a2021.0177 \\ Received: \\ September 6, 2021 \\ Revision accepted: \\ December 11, 2021 \\ Published online: \\ December 11, 2021
}

\section{A B S TR A C T}

Background: The highest rate of death is in the first few weeks after myocardial infarction (MI). However, the assessment of indications for primary prevention implantable cardioverter-defibrillator (ICD) implantation should be postponed until at least 40 days after MI.

Aims: Our aim was to identify the subgroup of high-risk patients with reduced left ventricular ejection fraction (LVEF) who would benefit from primary prevention ICD implantation within 40 days of MI.

Methods: Out of 205606 patients with MI, in this study, we included 18736 patients treated invasively, with $L V E F<40 \%$, who survived until hospital discharge. Patients were divided into two groups according to the survival status at 40 days - patients who died within this period $(n=1331)$ and patients who survived $(n=17405)$.

Results: Among all patients who died within 12-months after Ml, 37.7\% did die during the first 40 days. Patients with cardiac arrest before hospital admission or within the first 48 hours of hospitalization (hazard ratio [HR], 3.35; 95\% confidence interval [Cl], 2.82-3.98; $P<0.0001$ ], cardiogenic shock before admission or during hospitalization ( $\mathrm{HR}, 3.06 ; 95 \% \mathrm{Cl}, 2.62-3.59 ; P<0.0001)$, unsuccessful percutaneous coronary interventions ( $\mathrm{PCl} ; \mathrm{HR}, 2.42 ; 95 \% \mathrm{Cl}, 2.11-2.84 ; P<0.0001)$, LVEF $<20 \%$ (ref. LVEF $\geq 30 \% ; \mathrm{HR}, 2.75 ; 95 \% \mathrm{Cl}, 2.25-3.36 ; P<0.0001$ ) had approximately threefold and patients with chronic kidney disease almost 1.5-times $(\mathrm{HR}, 1.25 ; 95 \% \mathrm{Cl}, 1.47-3.59 ; P=0.0053)$ higher 40-day mortality compared to patients without these risk factors. The most striking differences in mortality between these subgroups were observed shortly after discharge.

Conclusions: The highest risk of death in patients with reduced LVEF who survived until hospital discharge occurred within the first 40 days after MI. There is a possibility to select patients with the worst prognosis and treat them more aggressively.

Key words: implantable cardioverter-defibrillator, myocardial infarction

Kardiol Pol 2021; 79, 12: 1343-1352 


\section{WHAT'S NEW?}

There is the highest mortality within the first 40 days after myocardial infarction (MI). In our study, of all patients who did not survive the first year after acute MI, 37.7\% died during the first 40 days. It means that one-third of patients die before LVEF reassessment and determining the indications for implantable cardioverter-defibrillator (ICD) or cardiac resynchronization therapy defibrillator (CTR-D) implantation in primary prevention. In our cohort, patients with MI were at approximately threefold higher risk of 40-day mortality if they had (1) cardiac arrest before or within the first 48 hours of admission; (2) cardiogenic shock; (3) unsuccessful percutaneous coronary intervention (final thrombolysis in myocardial infarction [TIMI], 0-2 flow) and left ventricular ejection fraction (LVEF) $<20 \%$ (compared to LVEF $\geq 30 \%$ ). Furthermore, the risk of death in patients with LVEF between 20\% and 30\% compared to patients with LVEF >30\% and patients aged over 65 years compared to younger ones was almost twice as high. Moreover, the risk of 40-day death in patients with chronic kidney disease was almost 1.5-times higher than other MI patients. The most significant differences in mortality between groups with and without the above-mentioned risk factors were observed in the short time after discharge.

\section{INTRODUCTION}

Over the last two decades, there has been a significant increase in the number of implantable cardioverter-defibrillator (ICD) implantation procedures. It has been proven that this therapy reduces mortality in patients with heart failure [1]. The current European Society of Cardiology (ESC) guidelines recommend ICD implantation as the standard treatment of patients with reduced left ventricular ejection fraction (LVEF) [2]. However, in patients who have recently had a myocardial infarction (MI), the assessment of indications for primary prevention ICD implantation should be postponed until at least 40 days after the acute coronary syndrome [3]. The IRIS (The Immediate Risk Stratification Improves Survival) and DINAMIT (the Defibrillator in Acute Myocardial Infarction Trial) studies showed that ICD implantation does not reduce all-cause mortality in this period but decreases the rate of death due to arrhythmia. However, the latter is offset by an increase in nonarrhythmic mortality $[4,5]$. According to the ESC guidelines, primary prevention ICD implantation in this period should be considered in case of incomplete revascularization and reduced LVEF prior to MI [3]. Despite the high prevalence of the invasive management strategy in the treatment of acute coronary syndromes, which has led to an improvement in in-hospital outcomes, long-term mortality in post-MI patients is still unsatisfactory, especially in those with reduced LVEF. It has been shown that $7 \%$ of patients with MI who survive until hospital discharge die or experience nonfatal cardiac arrest within 6 months. Additionally, in the subgroup of MI patients with LVEF lower than $30 \%$, the risk of death significantly increases during the first month after the event [6]. It must be noted that the most frequent cause of death in this group is ventricular arrhythmia [7]. Therefore, considering the highest rate of death and causes of death in the first few weeks after MI, we aimed to identify the group of high-risk patients with reduced LVEF who would benefit from primary prevention ICD implantation within 40 days of $\mathrm{MI}$.

\section{METHODS}

\section{SILCARD, PL-ACS, AMI-PL}

In the present study, we analyzed data from $205606 \mathrm{MI}$ patients (both ST-segment elevation and non-ST-segment elevation myocardial infarction) derived from three combined medical registries: SILCARD (The Silesian Cardiovascular Database), PL-ACS (Polish Registry of Acute Coronary Syndromes), and AMI-PL (Acute Myocardial Infarction in Poland).

SILCARD was developed under the agreement between the Silesian Center for Heart Disease in Zabrze and the Regional Department of National Health Fund (NHF) in Katowice, Poland to conduct epidemiological analyzes and prepare scientific elaborations on the group of patients with cardiovascular diseases (CVD) in the Silesia Province. The design and rationale of the SILCARD database were described previously [8]. The reported data come from 310 hospital wards and 1863 outpatient clinics and contain information on 487518 patients, 956634 cardiovascular hospitalizations, and 61906964 outpatient visits.

The PL-ACS is a clinically driven registry established in 2003, gathering detailed data on in-hospital management, treatment modalities, and outcomes. Its design and introduction were a joint effort of the Silesian Center for Heart Diseases in Zabrze and the Polish Ministry of Health. Currently, over 630000 hospitalizations for acute coronary syndrome are listed in the PL-ACS registry. Its detailed design has been described elsewhere [9].

The AMI-PL is a registry of administrative data gathered from all MI hospitalizations, containing data from the national healthcare provider. The database currently comprises information on over 550000 hospitalizations from 2009. The detailed design of AMI-PL has been presented previously [10].

According to letter no. PCN/0022/KB/49/21, keeping the register is not a medical experiment and does not require the consent of the ethics committee. 


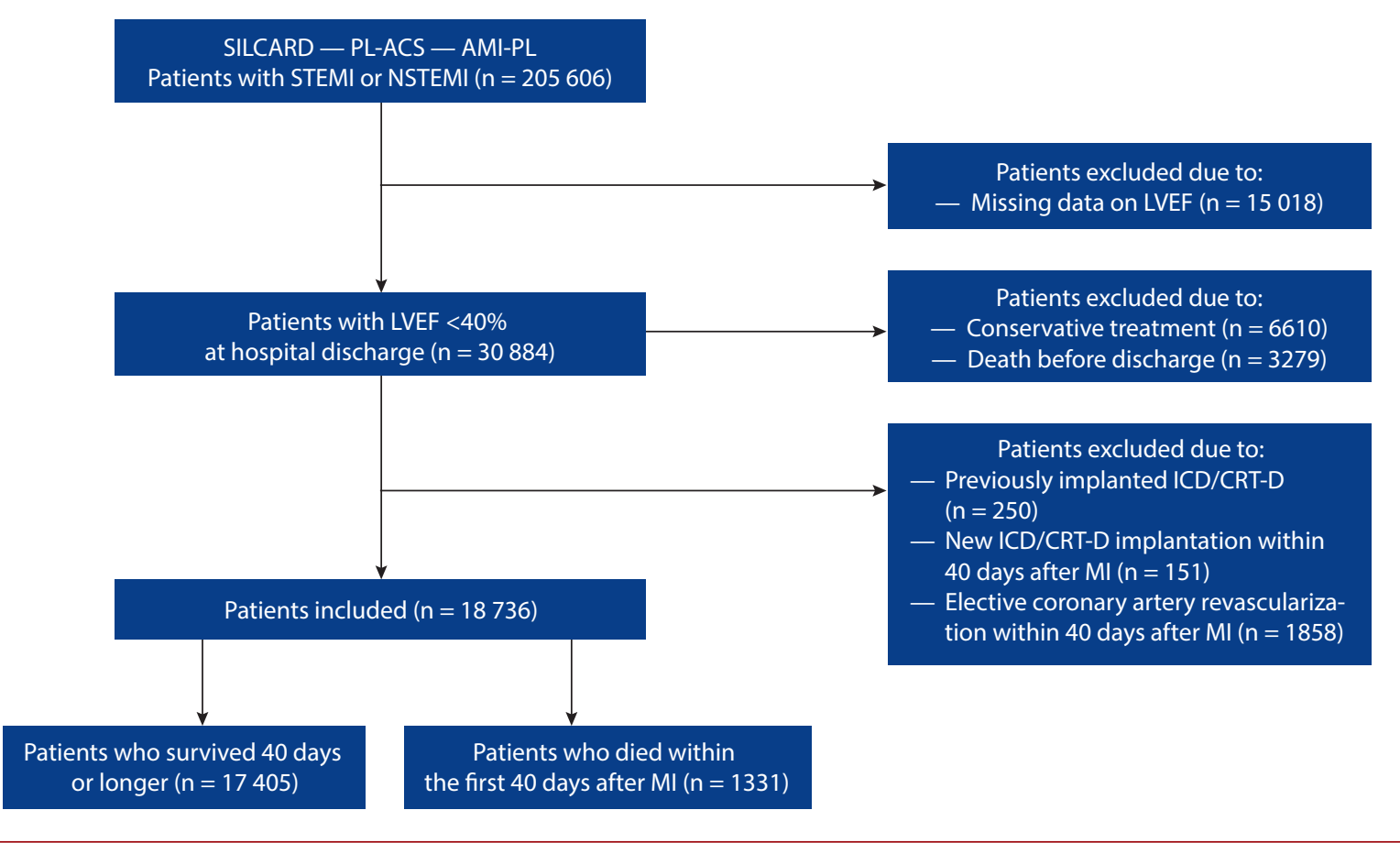

Figure 1. Flowchart of the study population

Abbreviations: AMI-PL, Acute Myocardial Infarction in Poland; CRT-D, cardiac resynchronization therapy defibrillator ICD, implantable cardioverter-defibrillator; LVEF, left ventricular ejection fraction; MI, myocardial infarction; PL-ACS, Polish Registry of Acute Coronary Syndromes; SILCARD, The Silesian Cardiovascular Database

\section{Study population}

A flowchart of the study population is shown in Figure 1. Patients who were included in the study were hospitalized with MI treated invasively, had LVEF $<40 \%$, and survived until hospital discharge. The exclusion criteria were as follows: MI treated with fibrinolysis or conservatively, ICD or cardiac resynchronization therapy defibrillator (CRT-D) implanted before the index hospitalization or within 40 days after MI, elective coronary artery revascularization within 40 days after MI, and missing data on LVEF. In total, 18736 patients were included. Patients were divided into two groups according to the survival status at 40 days - patients who died within this period $(n=1331)$ and patients who survived ( $n=17405)$. Selected clinical characteristics and angiographic parameters, as well as causes of rehospitalizations, were analyzed in both groups.

\section{Statistical analysis}

Qualitative parameters are presented as percentages while continuous parameters are presented as the median and interquartile range. Differences in categorical variables between the groups were tested using the $X^{2}$ test with Pearson modification, whereas differences in continuous variables were tested using the $U$ Mann-Whitney test. To assess the impact of particular parameters on 40-day mortality, a multivariable analysis was performed using step-down Cox proportional hazards regression modeling.
The multivariable model included the following variables: age, sex, LVEF, sudden cardiac arrest before admission or during hospitalization, cardiogenic shock, a history of arterial hypertension, diabetes mellitus, chronic kidney disease, tobacco smoking, diagnosis of ST-segment elevation myocardial infarction (STEMI), multivessel coronary artery disease, and final thrombolysis in myocardial infarction (TIMI) flow $0-2$ vs. 3 . The results of the multivariable analysis are expressed as a hazard ratio (HR) with the corresponding $95 \%$ confidence interval $(\mathrm{Cl})$. For mortality at 12 months, the analysis with the Kaplan-Meier method with the log-rank comparison of curves was performed. The level of statistical significance was $P<0.05$ (two-tailed). Statistica 10 software (StatSoft Inc., Tulsa, OK, USA) was used for all calculations.

\section{RESULTS}

\section{Clinical and angiographic characteristics}

Patients who died during a 40-day follow-up were older and more frequently female. Moreover, they were more likely to have cardiac arrest before admission or within the first 48 hours of hospital admission, as well as pulmonary edema, cardiogenic shock, hypertension, diabetes mellitus, a previous stroke, peripheral arterial disease, and had both lower blood pressure on admission and LVEF. Patients who died were less frequently current smokers and were less 
Table 1. Demographic and clinical characteristics of the study population

\begin{tabular}{|c|c|c|c|}
\hline & $\begin{array}{l}\text { Patients who survived } \\
40 \text { days }(n=17405)\end{array}$ & $\begin{array}{l}\text { Patients who died within } \\
40 \text { days }(n=1331)\end{array}$ & $P$-value \\
\hline Age, years & $69(60-77)$ & $74(65-80)$ & $<0.0001$ \\
\hline Male sex, $n(\%)$ & $11884(68.28)$ & $875(65.74)$ & 0.056 \\
\hline Hypertension, n (\%) & $12546(72.08)$ & $913(68.6)$ & 0.0064 \\
\hline Current or former smokers, $\mathrm{n}(\%)$ & $10201(58.61)$ & $720(54.11)$ & 0.0013 \\
\hline Type 2 diabetes mellitus, $\mathrm{n}(\%)$ & $5803(33.34)$ & $520(39.05)$ & $<0.0001$ \\
\hline Obesity, n (\%) & $3448(19.81)$ & $251(18.87)$ & 0.41 \\
\hline Previous MI, n (\%) & $5046(28.99)$ & $355(26.67)$ & 0.072 \\
\hline Previous PCl, n (\%) & $3483(20.01)$ & $211(15.85)$ & 0.0002 \\
\hline Previous CABG, $\mathrm{n}(\%)$ & $1046(6.01)$ & $56(4.21)$ & 0.007 \\
\hline Previous stroke, $\mathrm{n}(\%)$ & $870(5.0)$ & $132(9.95)$ & $<0.0001$ \\
\hline PAD, n (\%) & $1173(6.74)$ & $140(10.53)$ & $<0.0001$ \\
\hline STEMI, n (\%) & $9844(56.56)$ & $790(59.35)$ & 0.047 \\
\hline Atrial fibrillation on admission, $\mathrm{n}(\%)$ & $1558(8.95)$ & $34(2.53)$ & 0.001 \\
\hline Diabetes mellitus, $\mathrm{n}(\%)$ & $5803(33.34)$ & $520(39.05)$ & $<0.0001$ \\
\hline Chronic kidney disease, $\mathrm{n}(\%)$ & $1897(10.90)$ & $233(17.48)$ & $<0.0001$ \\
\hline Systolic blood pressure on admission, $\mathrm{mm} \mathrm{Hg}$ & $130(105-147)$ & $120(100-140)$ & $<0.0001$ \\
\hline Diastolic blood pressure on admission, $\mathrm{mm} \mathrm{Hg}$ & $80(70-100)$ & $77(65-80)$ & $<0.0001$ \\
\hline Heart rate, $1 / \mathrm{min}$ & $80(70-100)$ & $90(75-100)$ & $<0.0001$ \\
\hline LVEF, \% & $31(27-35)$ & $30(20-35)$ & $<0.0001$ \\
\hline $\begin{array}{l}\text { Sudden cardiac arrest before admission or within the first } 48 \text { hours } \\
\text { of hospital admission, } n(\%)\end{array}$ & $599(3.44)$ & $254(19.1)$ & $<0.0001$ \\
\hline Pulmonary edema, $\mathrm{n}(\%)$ & $1083(6.22)$ & $150(11.24)$ & $<0.0001$ \\
\hline Cardiogenic shock, $\mathrm{n}(\%)$ & $731(4.2)$ & $220(16.52)$ & $<0.0001$ \\
\hline
\end{tabular}

Continuous variables are presented as median (interquartile range [IQR]). Dichotomous variables are presented as percentages

Abbreviations: CABG, coronary artery bypass grafting; PAD, peripheral artery disease; PCl, percutaneous coronary intervention; STEMI, ST-elevation myocardial infarction; other - see Figure 1

Table 2. Angiographic characteristics and in-hospital outcomes

\begin{tabular}{|c|c|c|c|}
\hline & $\begin{array}{c}\text { Patients who survived } \\
40 \text { days } \\
(n=17405)\end{array}$ & $\begin{array}{l}\text { Patients who died within } \\
40 \text { days } \\
(n=1331)\end{array}$ & P-value \\
\hline \multicolumn{4}{|l|}{ Infarct-related artery, n (\%) } \\
\hline Left main & $573(3.29)$ & $75(5.64)$ & $<0.0001$ \\
\hline Left anterior descending & $9031(51.89)$ & $687(51.65)$ & \\
\hline Diagonal branch & $350(2.02)$ & $23(1.73)$ & \\
\hline Circumflex & $2273(13.06)$ & $178(13.38)$ & \\
\hline Obtuse marginal branch & $714(4.1)$ & $53(3.98)$ & \\
\hline Right coronary artery & $3754(21.57)$ & $271(20.38)$ & \\
\hline Saphenous vein or arterial graft & $343(1.97)$ & $20(1.5)$ & \\
\hline Not determined & $366(2.1)$ & $23(1.74)$ & \\
\hline Multivessel coronary artery disease, $\mathrm{n}(\%)$ & $10956(62.95)$ & $930(69.9)$ & 0.0044 \\
\hline $\mathrm{PCl}, \mathrm{n}(\%)$ & 17351 (99.69) & $1,327(99.7)$ & 0.95 \\
\hline \multicolumn{4}{|l|}{ Final TIMI flow, n (\%) } \\
\hline 0 & $517(2.97)$ & $180(13.49)$ & $<0.0001$ \\
\hline 1 & $261(1.5)$ & $58(4.33)$ & \\
\hline 2 & $896(5.15)$ & $144(10.82)$ & \\
\hline 3 & 15732 (90.39) & $951(71.46)$ & \\
\hline CABG, $n(\%)$ & $54(0.31)$ & $4(0.3)$ & 0.94 \\
\hline Inotropes or vasopressors, n (\%) & $898(5.16)$ & $253(19.01)$ & $<0.0001$ \\
\hline IABP, n (\%) & $369(2.12)$ & $143(10.74)$ & $<0.0001$ \\
\hline Reinfarction, n (\%) & $101(0.58)$ & $19(1.43)$ & 0.0002 \\
\hline Major bleeding, n (\%) & $190(1.09)$ & $41(3.08)$ & $<0.0001$ \\
\hline
\end{tabular}

Abbreviations: IABP, intra-aortic balloon pump; other — see Table 1

likely to have previous coronary artery revascularization in comparison with individuals who survived 40 days (Table 1). In the group of patients who died, there was a higher percentage of patients with multivessel disease and the left main artery as an infarct-related artery. Patients who survived 40 days had more likely the left anterior descending artery as an infarct-related artery and a higher success rate of percutaneous coronary intervention (PCI) (Table 2). The success rate in the whole study population (TIMI 3 flow after PCI) was $89.03 \%$. 
Table 3. Pharmacological treatment at discharge

\begin{tabular}{|c|c|c|c|}
\hline & $\begin{array}{l}\text { Patients who survived } \\
40 \text { days }(n=17405)\end{array}$ & $\begin{array}{l}\text { Patients who died within } \\
40 \text { days }(n=1331)\end{array}$ & $P$-value \\
\hline ASA, n (\%) & $16738(96.17)$ & 1239 (93.09) & $<0.0001$ \\
\hline Clopidogrel, n (\%) & 15765 (90.58) & $1154(86.7)$ & $<0.0001$ \\
\hline ACE-I or ARB, n (\%) & 14477 (83.18) & $827(62.13)$ & $<0.0001$ \\
\hline$\beta$-blocker, n (\%) & 15365 (88.28) & $932(70.02)$ & $<0.0001$ \\
\hline Statin, n (\%) & $16117(92.6)$ & $1103(82.9)$ & $<0.0001$ \\
\hline Nitrate, n (\%) & $2277(13.08)$ & $145(10.89)$ & 0.022 \\
\hline LMWH, n (\%) & $943(5.42)$ & $180(13.52)$ & $<0.0001$ \\
\hline Oral anticoagulant, $\mathrm{n}(\%)$ & 809 (4.65) & $34(2.53)$ & 0.001 \\
\hline VKA & $727(89.86)$ & $23(67.65)$ & 0.0001 \\
\hline NOAC & $82(10.14)$ & $11(32.35)$ & \\
\hline
\end{tabular}

Abbreviations: ASA, acetylsalicylic acid; ACE-I, angiotensin-converting-enzyme inhibitor; ARB, angiotensin II receptor blocker; LMWH, low-molecular-weight heparin; NOAC, non-VKA oral anticoagulant; VKA, vitamin-K antagonist

\section{In-hospital treatment and outcomes and prescribed medicines at discharge}

In patients who died in 40 days following MI, major bleeding and recurrent MI were more often observed. These patients were more likely to require inotropic support and intra-aortic balloon pumps (Table 2). Moreover, they were less likely to be prescribed antiplatelets, $\beta$-blockers, ACE inhibitors/angiotensin II receptor antagonists, statins, and nitrates. On the other hand, patients who survived 40 days were less frequently discharged on anticoagulants (Table 3).

\section{Forty-day mortality and its predictors}

Forty-day and 1-year mortality in patients with $\mathrm{MI}$ and LVEF $<40 \%$ who survived until hospital discharge was $7.1 \%$ and $18.81 \%$, respectively. The cumulative mortality rate in this group is shown in Figure 2. In the multivariable analysis, the independent predictors of 40-day mortality were as follows: cardiac arrest before hospital admission or within the first 48 hours of hospitalization, cardiogenic shock before admission or during hospitalization, unsuccessful $\mathrm{PCl}$, lower LVEF, age $>65$ years, and chronic kidney disease (Table 4). Cumulative mortality rate curves for patients stratified according to independent predictors of death are depicted in Figures 2 and 3. We found ca. three-fold higher 40-day mortality in patients with cardiac arrest, cardiogenic shock, or unsuccessful percutaneous coronary intervention compared to patients without these complications.

\section{Rehospitalizations within $\mathbf{4 0}$ days}

There were significantly higher overall and cardiovascular 40-day readmission rates in patients who died during this period. Cardiac arrest was significantly more frequent, while stable coronary artery disease and unstable angina were significantly less frequent causes of readmission in this group, compared to patients who survived 40 days (Table 5). The median time from hospital discharge to readmission in patients who died and survived 40 days was 10 (5.25-18) and 24 (14-33) days, respectively.

\section{DISCUSSION}

In the present study patients with $\mathrm{MI}$ and reduced LVEF were analyzed. This group included $14.5 \%$ of all patients with Ml, who had survived the in-hospital period. This percentage was slightly lower than described in other studies, i.e. $21.4 \%$ in the study by Zaman et al. [11]. The above-mentioned discrepancies might stem from the fact that in the present analysis, patients treated conservatively and patients with implanted ICD or CRT-D were excluded. Nevertheless, it is worth emphasizing, that every seventh patient with MI had LVEF $<40 \%$ at hospital discharge. The ESC guidelines suggest a subsequent reassessment of LVEF 40 days after $\mathrm{Ml}$ in these patients. After this period further therapeutic decisions should be made. Considering the results of our study, it seems that this recommendation should be re-examined. In our study, we showed, that approximately $38 \%$ of deaths in the first year after Ml occur within the first 40 days. It means that one-third of patients die within the first year of MI before LVEF reassessment and starting adequate treatment, which could affect the survival of this group of patients. Thus, it seems reasonable to re-evaluate whether there is a subgroup of patients who should undergo ICD or CRT-D implantation in primary prevention before hospital discharge or shortly after MI.

To the best of our knowledge, there is no clear solution to the problem in the available literature. Randomized clinical trials assessing ICD effectiveness for primary prevention of sudden cardiac death within the first 40 days after MI have shown unambiguously no significant survival benefit from ICD implantation over optimal medical therapy. The way of randomization and timing of the procedure in these studies is worth analyzing. Patients recruited to the IRIS trial had acute MI 5-31 days before randomization. Approximately $7 \%$ of patients did not undergo ICD implantation despite allocation to the ICD group. Moreover, $9 \%$ of patients were discharged from the hospital before the planned procedure, and $1 \%$ of patients died before ICD implantation [4]. The inclusion criteria for the DINAMIT trial were acute MI 6-40 days before enrollment (18 days on average) and the possibility of ICD implantation within 




Figure 2. A. Kaplan-Meier curves for patients who died within 1 year of myocardial infarction. B. Kaplan-Meier curves for patients with and without cardiac arrest before admission or within the first 48 hours of hospitalization. C. Kaplan-Meier curves for patients with and without cardiogenic shock before admission or during hospitalization

Table 4. Predictors for 40-day mortality — a multivariable analysis

\begin{tabular}{|c|c|c|}
\hline & HR $(95 \%$ Cl) & $P$-value \\
\hline Sudden cardiac arrest before admission or within the first 48 hours of hospital admission & $3.35(2.82-3.98)$ & $<0.0001$ \\
\hline Unsuccessful PCI & $2.42(2.11-2.84)$ & $<0.0001$ \\
\hline Cardiogenic shock before admission or during hospitalization & $3.06(2.62-3.59)$ & $<0.0001$ \\
\hline LVEF $<20 \%{ }^{a}$ & $2.75(2.25-3.36)$ & $<0.0001$ \\
\hline LVEF $20 \%-30 \%^{\mathrm{a}}$ & $1.90(1.68-2.16)$ & $<0.0001$ \\
\hline Age $>65$ years & $1.82(1.59-2.07)$ & $<0.0001$ \\
\hline Chronic kidney disease & $1.25(1.07-1.47)$ & 0.0053 \\
\hline
\end{tabular}

aVs. LVEF $\geq 30 \%$

Abbreviations: $\mathrm{Cl}$, confidence interval; $\mathrm{HR}$, hazard ratio; other — see Table 1 and Figure 1 



Figure 3. A. Kaplan-Meier curves for patients stratified by final TIMI flow. B. Kaplan-Meier curves for patients stratified by left ventricular ejection fraction. C. Kaplan-Meier curves for patients stratified by age. D. Kaplan-Meier curves for patients with and without CKD Abbreviations: CKD, chronic kidney disease, other — see Table 4

Table 5. 40-day readmissions and procedures during follow-up

\begin{tabular}{|c|c|c|c|}
\hline & $\begin{array}{l}\text { Patients who survived } \\
40 \text { days }(n=17405)\end{array}$ & $\begin{array}{l}\text { Patients who died within } \\
40 \text { days }(n=1331)\end{array}$ & $P$-value \\
\hline Readmission rate, $\mathrm{n}(\%)$ & $3563(20.47)$ & $695(52.25)$ & $<0.0001$ \\
\hline Readmission for cardiovascular disease, $\mathrm{n}(\%)$ & $3084(17.72)$ & 617 (46.38) & $<0.0001$ \\
\hline \multicolumn{4}{|l|}{ Causes of readmissions, $\mathrm{n}(\%)$} \\
\hline Atrial fibrillation & $75(2.43)$ & $8(1.32)$ & \multirow{12}{*}{$<0.0001$} \\
\hline Supraventricular arrhythmias & $25(0.81)$ & $3(0.44)$ & \\
\hline Ventricular arrhythmias (except for sudden cardiac death) & $19(0.61)$ & $2(0.32)$ & \\
\hline Conduction disorders & $35(1.13)$ & $11(1.76)$ & \\
\hline Sudden cardiac arrest & $18(0.59)$ & $68(11.02)$ & \\
\hline Pulmonary embolism & $14(0.45)$ & $5(0.88)$ & \\
\hline Valvular heart disease & $110(3.56)$ & $16(2.64)$ & \\
\hline Heart failure & $1130(36.64)$ & $239(38.77)$ & \\
\hline Myocardial infarction & $230(7.46)$ & $60(9.69)$ & \\
\hline Unstable angina & $366(11.87)$ & $19(3.08)$ & \\
\hline Stable coronary artery disease & $897(29.09)$ & $73(11.89)$ & \\
\hline Other & $165(5.36)$ & $112(18.19)$ & \\
\hline \multicolumn{4}{|l|}{ Procedures performed during follow-up, $\mathrm{n}(\%)$} \\
\hline Coronary angiography & $2026(11.64)$ & $11(0.81)$ & $<0.001$ \\
\hline $\mathrm{PCl}$ & $1004(5.77)$ & $2(0.15)$ & $<0.0001$ \\
\hline CABG & $132(0.76)$ & $0(0)$ & 0.27 \\
\hline Cardiac ablation & $38(0.22)$ & $0(0)$ & 0.55 \\
\hline
\end{tabular}

Abbreviations - see Table 1 
7 days of randomization. Approximately $6 \%$ of patients allocated to the ICD group did not undergo device implantation. The mean time between randomization and the procedure was $6.3 \pm 7.3$ days [5]. It should be noted that in both studies, survival analysis was conducted from the moment of randomization and not from the MI [4, 5]. The Multicenter Automatic Defibrillator Implantation Trial (MADIT)-II, based on which the ESC recommends ICD implantation at least 40 days after Ml, showed a significant reduction of overall mortality compared to optimal medical therapy only after approximately 9 months of ICD therapy. It is noteworthy that $88 \%$ of patients who underwent ICD implantation were included in the study $>6$ months after MI [1]. It appears from the above data that patients included in the trials, on which ESC guidelines were based, did not reflect the "real-world" population. Some patients may have died before recruitment or between randomization and ICD implantation. Nowadays, in the era of interventional treatment of $\mathrm{Ml}$, patients are discharged from the hospital early (usually on the $4^{\text {th }}$ or $5^{\text {th }}$ day), and the early start post-hospital period is characterized by a high risk of death in some patients. In our study, the eligibility criteria analogous to those for randomized clinical trials regarding ICD implantation after MI were not applied. Elimination of these limitations should enable researchers to establish the role of primary prevention ICD implantation in reducing early sudden cardiac deaths after MI.

Over the last few years, wearable cardioverter-defibrillators (WCDs) have emerged as a bridge to ICD implantation in patients at risk of sudden cardiac death soon after MI. A recent randomized clinical trial (Vest Prevention of Early Sudden Death Trial [VEST]) included patients with acute myocardial infarction and LVEF of $35 \%$ or less. It has been shown that there was no difference in arrhythmic deaths (sudden death or death from ventricular tachyarrhythmia) between participants assigned to receive a WCD and the control group. However, there was significantly lower allcause mortality in the device group. It should be pointed out that patients were enrolled up to 7 days after hospital discharge and participants in the device group wore the device, on average, only 14 hours a day. Of the 48 patients in this group, 36 patients who died were not wearing the device at the time of the incident. These facts may explain the negative results of the VAST trial. Nonetheless, data from the trial confirm that the highest risk of arrhythmic death occurs during the first 30 days after MI. Therefore, in certain patients, WCDs might reduce mortality if they are worn for the recommended time per day [12]. In 2019, experts of the Heart Rhythm Section of the Polish Cardiac Society gave their opinion on the use of WCDs in Poland. They suggest that up to three months after MI, WCD therapy can be considered in patients with a history of sustained VT orVF during the first 48 hours of ACS, cardiogenic shock or pulmonary edema, and with a history of asymptomatic permanent VT [13]

\section{Forty-day mortality}

In our study, we showed that 40-day mortality after MI is still very high (during this period occurs approximately one-third of all deaths within the first year). Our results are consistent with previous reports. In the VALsartan In Acute myocardial iNfarcTion (VALIANT) trial, the highest risk of death was within the first week after MI. During the first month of $\mathrm{Ml}$, the risk of cardiovascular death or nonfatal cardiac arrest was $1.4 \%$, but between months 6 and 12 , the risk declined to $0.27 \%$ per month [6]. We found independent predictors of 40-day mortality, which enabled us to identify patients with a greater risk of death early after MI.

\section{Sudden cardiac arrest}

In our analysis, the strongest predictor of early death was sudden cardiac arrest before admission or within the first $48 \mathrm{~h}$ of hospital admission. The risk of death early after hospital discharge in this group was 3-times higher compared to patients without cardiac arrest. Other studies have not confirmed this finding. It has been shown that patients with MI complicated by out-of-hospital cardiac arrest who are alive at hospital discharge have a similar risk of death compared to the other MI patients [14, 15]. This might be the reason for the abovementioned discrepancies. To the best of our knowledge, there are no studies that aimed to analyze the relationship between in-hospital cardiac arrest and early mortality after discharge in a population similar to our cohort.

\section{Unsuccessful PCI}

In the present study, we showed that patients with unsuccessful PCI had almost 3-fold higher 40-day mortality compared to subjects with final TIMI flow 3 in the infarct-related artery. The multivariable analysis indicated that unsuccessful PCI was an independent predictor of death. Unsuccessful PCl leads to greater myocardial injury and, as a consequence, to reduced LVEF. This, in turn, results in an increased risk of serious life-threatening arrhythmias. These findings have been previously reported [16].

\section{Cardiogenic shock}

Cardiogenic shock complicating acute $\mathrm{Ml}$ is associated with a less favorable prognosis. Shah et al. [17] evaluated the relationship between cardiogenic shock and mortality in patients who were alive at hospital discharge. They found that the highest risk of death occurred within the first 60 days after hospital discharge. During the 40-day follow-up, mortality was approximately 1.5-times higher compared to patients without cardiogenic shock. The FAST-MI (French Registry on Acute ST-elevation and non-ST-elevation Myocardial Infarction) study provided similar results [18]. Our results are consistent with these reports. We showed that in Ml patients with cardiogenic shock, the highest mortality occurs within 40 days following MI. The reason for this might be the fact that patients who develop cardiogenic 
shock are older, have more comorbidity burden, lower $\mathrm{LVEF}$, more frequently unsuccessful $\mathrm{PCl}$, and more frequent out-of-hospital and in-hospital sudden cardiac arrest than patients without this complication $[17,18]$.

\section{Left ventricular ejection fraction}

Left ventricular ejection fraction is an important predictor of mortality in patients with MI. Margolis et al. analyzed the LVEF of 2086 STEMI patients assessed within 72 hours of episode onset. Patients with LVEF lower than $40 \%$ had approximately 6-times higher 30-day mortality than those with LVEF $>40 \%$ [19]. In the VALIANT study, Ml patients with $L V E F<40 \%$ or radiological evidence of heart failure were included. The rate of sudden cardiac death, including resuscitated sudden cardiac death, at 30 days was $2.3 \%$ and less than $1 \%$ in patients with LVEF $\leq 30 \%$ and $>30 \%$, respectively. The risk of major adverse cardiovascular events increased by $21 \%$ for each $5 \%$ decrease in LVEF [6]. Similarly, the present study demonstrated that reduced LVEF was associated with higher 40-day mortality.

\section{Age}

In our study, we observed that patients' age was an independent predictor of death within 40 days. The relative risk of death in patients aged over 65 years was almost twice as high as in younger patients. This may be due to a linear relationship between mortality after $\mathrm{Ml}$ and age. This risk increases significantly for each subsequent year of age [19].

\section{Rehospitalizations in the 40-day follow-up}

Most rehospitalizations in patients with Ml occur early after hospital discharge. Kim et al. [20] reported that two-thirds of readmissions in STEMI patients occurred up to 14 days of hospital discharge. The median time to readmission in this study was 9 days. The readmission rate for Ml or heart failure was higher in this period compared to subsequent periods. Additionally, the MINAP registry (Myocardial Ischemia National Audit Project) provided similar findings [21]. In the present study, the 40 -day readmission rate was $22 \%$, which was higher than in other studies $[20,21]$. The reason might be the fact that only patients with LVEF $<40 \%$ were included in our study. In our analysis, heart failure was the most frequent cause of readmission, followed by acute coronary syndromes. Moreover, patients who died up to 40 days after MI were significantly more frequently readmitted for sudden cardiac arrest than patients who survived. We had no detailed data about the mechanism of sudden cardiac death. However, considering epidemiological data regarding patients with left ventricular systolic dysfunction after MI, we can suspect that most of them died of ventricular fibrillation or pulseless ventricular tachycardia. Therefore, it seems that primary prevention ICD implantation might reduce 40 -day mortality in these patients.

\section{Limitations}

The data on causes of readmission within 40 days were derived from an administrative database, which has some limitations [22]. According to the ICD-10 classification, the principal diagnosis reported to the National Health Fund most often reflects the real reason for hospitalization. However, the reporting systems are not standardized. For example, the order and number of diagnoses are subjectively reported, therefore, some disease entities can be omitted. Moreover, the classification often does not determine the subcategories of individual diseases, which hinders the precise determination of all the diagnoses.

Another limitation of the study might be the lack of information on the causes of death in all patients. We would like to emphasize that we report all-cause mortality, not cardiac mortality. However, given the short time from discharge to death, it can be assumed with a high probability that these were sudden cardiac deaths and not consequences of chronic diseases.

\section{CONCLUSIONS}

The highest risk of death in patients with reduced LVEF occurs within 40 days after MI. These deaths constitute one-third of all deaths that occur during the first year. The development of an optimal treatment strategy and possible ICD implantation during this period, in a certain group of patients, can be another step toward reducing mortality.

\section{Article information}

Conflict of interest: None declared.

Open access: This article is available in open access under Creative Common Attribution-Non-Commercial-No Derivatives 4.0 International (CC BY-NC-ND 4.0) license, allowing to download articles and share them with others as long as they credit the authors and the publisher, but without permission to change them in any way or use them commercially. For commercial use, please contact the journal office at kardiologiapolska@ptkardio.pl.

How to cite: Pres D, Tajstra M, Mitkowski P, et al. Prediction of early death after myocardial infarction in patients with reduced left ventricular ejection fraction. The search for new indications for cardioverter-defibrillator implantation. Kardiol Pol. 2021; 79(12): 1343-1352, doi: 10.33963/KP.a2021.0177.

\section{REFERENCES}

1. Moss AJ, Zareba W, Hall WJ, et al. Prophylactic implantation of a defibrillator in patients with myocardial infarction and reduced ejection fraction. N Engl J Med. 2002; 346(12): 877-883, doi: 10.1056/NEJMoa013474, indexed in Pubmed: 11907286.

2. Ponikowski P, Voors A, Anker S, et al. 2016ESC Guidelines for the diagnosis and treatment of acute and chronic heart failure. Eur J Heart Fail. 2016; 18(8): 891-975, doi: 10.1002/ejhf.592, indexed in Pubmed: 27207191.

3. Priori SG, Blomström-Lundqvist C, Mazzanti A, et al. 2015 ESC Guidelines for the management of patients with ventricular arrhythmias and the prevention of sudden cardiac death: The Task Force for the Management of Patients with Ventricular Arrhythmias and the Prevention of Sudden Cardiac Death of the European Society of Cardiology (ESC). Endorsed by: Association for European Paediatric and Congenital Cardiology (AEPC). Eur Heart J. 2015; 36(41): 2793-2867, doi: 10.1093/eurheartj/ehv316, indexed in Pubmed: 26320108. 
4. Steinbeck G, Andresen D, Seidl K, et al. Defibrillator implantation early after myocardial infarction. N Engl J Med. 2009; 361(15): 1427-1436, doi: 10.1056/NEJMoa0901889, indexed in Pubmed: 19812399.

5. Hohnloser $\mathrm{SH}$, Kuck KH, Dorian P, et al. Prophylactic use of an implantable cardioverter-defibrillator after acute myocardial infarction. N Engl J Med. 2004; 351(24): 2481-2488, doi: 10.1056/NEJMoa041489, indexed in Pubmed: 15590950.

6. Solomon SD, Zelenkofske S, McMurray J. Sudden death in patients with myocardial infarction and left ventricular dysfunction, heart failure, or both. N Engl J Med. 2005; 352(25): 2581-2588, doi: 10.1056/NEJMoa043938, indexed in Pubmed: 15972864.

7. Jordaens L, Tavernier R. Determinants of sudden death after discharge from hospital for myocardial infarction in the thrombolytic era. Eur Heart J. 2001; 22(14): 1214-1225, doi: 10.1053/euhj.2000.2464, indexed in Pubmed: 11440494.

8. Gąsior M, Pres D, Wojakowski W, et al. Causes of hospitalization and prognosis in patients with cardiovascular diseases. Secular trends in the years 2006-2014 according to the SILesian CARDiovascular (SILCARD) database. Pol Arch Med Wewn. 2016; 126(10): 754-762, doi: 10.20452/pamw.3557, indexed in Pubmed: 27650214.

9. Poloński L, Gasior M, Gierlotka M, et al. Polish Registry of Acute Coronary Syndromes (PL-ACS). Characteristics, treatments and outcomes of patients with acute coronary syndromes in Poland. Kardiol Pol. 2007; 65(8):861-72; discussion 873, indexed in Pubmed: 17853315.

10. Wojtyniak B, Gierlotka M,Opolski G, et al. Incidence, treatment, in-hospital mortality and one-year outcomes of acute myocardial infarction in Poland in 2009-2012--nationwide AMI-PL database. Kardiol Pol. 2015; 73(3): 142-158, doi: 10.5603/KP.a2014.0213, indexed in Pubmed: 25371307.

11. Zaman S, Sivagangabalan G, Narayan A, et al. Outcomes of early risk stratification and targeted implantable cardioverter-defibrillator implantation after ST-elevation myocardial infarction treated with primary percutaneous coronary intervention. Circulation. 2009; 120(3): 194-200, doi: 10.1161/CIRCULATIONAHA.108.836791, indexed in Pubmed: 19581496.

12. Lee BK, Pletcher MJ, Olgin JE, et al. Wearable cardioverter-defibrillator after myocardial infarction. N Engl J Med. 2018; 379(13): 1205-1215, doi: 10.1056/NEJMoa1800781, indexed in Pubmed: 30280654.

13. Sterliński M, Oręziak A, Przybylski A, et al. Experts of the Heart Rhythm Section of the Polish Cardiac Society: opinion on the use of wearable cardioverter-defibrillators in Poland. Kardiol Pol. 2019; 77(2): 238-243, doi: 10.5603/KP.2019.0031, indexed in Pubmed: 30816992
14. Demirel F, Rasoul S, Elvan A, et al. Impact of out-of-hospital cardiac arrest due to ventricular fibrillation in patients with ST-elevation myocardial infarction admitted for primary percutaneous coronary intervention: Impact of ventricular fibrillation in STEMI patients. Eur Heart J Acute Cardiovasc Care. 2015; 4(1): 16-23, doi: 10.1177/2048872614547448, indexed in Pubmed: 25114328.

15. Liang JJ, Fender EA, Cha YM, et al. Long-Term Outcomes in Survivors of Early Ventricular Arrhythmias After Acute ST-Elevation and Non-ST-Elevation Myocardial Infarction Treated With Percutaneous Coronary Intervention. Am J Cardiol. 2016; 117(5): 709-713, doi: 10.1016/j.amjcard.2015.12.002, indexed in Pubmed: 26796195.

16. Karwowski J, Poloński L, Gierlotka M, et al. Post-procedural TIMI flow grade 2 is not associated with improved prognosis in patients with non-ST-segment elevation myocardial infarction undergoing percutaneous coronary revascularization (PL-ACS registry). Cardiol J. 2016; 23(4): 402-410, doi: 10.5603/CJ.a2016.0025, indexed in Pubmed: 27296160.

17. Shah RU, de Lemos JA, Wang TY, et al. Post-Hospital outcomes of patients with acute myocardial infarction with cardiogenic shock: findings from the NCDR. J Am Coll Cardiol. 2016; 67(7): 739-747, doi: 10.1016/j. jacc.2015.11.048, indexed in Pubmed: 26892407.

18. Aissaoui N, Puymirat E, Simon T, et al. Long-term outcome in early survivors of cardiogenic shock at the acute stage of myocardial infarction: a landmark analysis from the French registry of Acute ST-elevation and non-ST-elevation Myocardial Infarction (FAST-MI) Registry. Crit Care. 2014; 18(5):516, doi: 10.1186/s13054-014-0516-y, indexed in Pubmed: 25246084.

19. Margolis G, Khoury S, Ben-Shoshan J, et al. Prognostic implications of midrange left ventricular ejection fraction on patients presenting with ST-segment elevation myocardial infarction. Am J Cardiol. 2017; 120(2): 186-190, doi: 10.1016/j.amjcard.2017.04.005, indexed in Pubmed: 28532775.

20. Kim LK, Yeo I, Cheung JW, et al. Thirty-Day readmission rates, timing, causes, and costs after st-segment-elevation myocardial infarction in the united states: a national readmission database analysis 2010-2014. J Am Heart Assoc. 2018; 7(18): e009863, doi: 10.1161/JAHA.118.009863, indexed in Pubmed: 30371187.

21. KwokCS, Wong CW, Shufflebotham $\mathrm{H}$, et al. Early readmissions after acute myocardial infarction. Am J Cardiol. 2017; 120(5): 723-728, doi: 10.1016/j. amjcard.2017.05.049, indexed in Pubmed: 28728745.

22. Johnson EK, Nelson CP. Values and pitfalls of the use of administrative databases for outcomes assessment. J Urol. 2013; 190(1): 17-18, doi: 10.1016/j.juro.2013.04.048, indexed in Pubmed: 23608038. 